\title{
Dopamine and Serotonin Are Both Required for Mate-Copying in Drosophila melanogaster
}

\author{
Magdalena Monier ${ }^{1 *}$, Sabine Nöbel ${ }^{1,2}$, Etienne Danchin ${ }^{1 \dagger}$ and Guillaume Isabel ${ }^{3 \dagger}$ \\ 1 Laboratoire Évolution \& Diversité Biologique, UMR5174, CNRS, IRD, Université Toulouse III - Paul Sabatier, Toulouse, \\ France, ${ }^{2}$ Institute for Advanced Study in Toulouse, Toulouse, France, ${ }^{3}$ Centre de Recherches sur la Cognition Animale \\ $(C R C A)$, Centre de Biologie Intégrative (CBI), Université de Toulouse, CNRS, UPS, Toulouse, France
}

\section{OPEN ACCESS}

Edited by:

Ellouise Leadbeater,

University of London, United Kingdom

Reviewed by:

Young-Joon Kim,

Gwangju Institute of Science and Technology, South Korea

Raúl G. Paredes,

National Autonomous University

of Mexico, Mexico

Divya Sitaraman,

University of San Diego, United States

*Correspondence:

Magdalena Monier

magdalena.monier@univ-tlse3.fr

${ }^{\dagger}$ Co-senior authors

Received: 29 June 2018 Accepted: 19 December 2018

Published: 09 January 2019

Citation:

Monier M, Nöbel S, Danchin E and Isabel G (2019) Dopamine and Serotonin Are Both Required for Mate-Copying in Drosophila

melanogaster.

Front. Behav. Neurosci. 12:334. doi: 10.3389/fnbeh.2018.00334
Mate-copying is a form of social learning in which the mate-choice decision of an individual (often a female) is influenced by the mate-choice of conspecifics. Drosophila melanogaster females are known to perform such social learning, and in particular, to mate-copy after a single observation of one conspecific female mating with a male of one phenotype, while the other male phenotype is rejected. Here, we show that this form of social learning is dependent on serotonin and dopamine. Using a pharmacological approach, we reduced dopamine or serotonin synthesis in adult virgin females with 3-iodotyrosine (3-IY) and DL-para-chlorophenylalanine (PCPA), respectively, and then tested their mate-copying performance. We found that, while control females without drug treatment copied the choice of the demonstrator, drug-treated females with reduced dopamine or serotonin chose randomly. To ensure the specificity of the drugs, the direct precursors of the neurotransmitters, either the dopamine precursor L-3,4dihydroxyphenylalanine (L-DOPA) or the serotonin precursor 5-L-hydroxytryptophan (5-HTP) were given together with the drug, (respectively 3-IY and PCPA) resulting in a full rescue of the mate-copying defects. This indicates that dopamine and serotonin are both required for mate-copying. These results give a first insight into the mechanistic pathway underlying this form of social learning in $D$. melanogaster.

Keywords: fruit fly, mate choice, social learning, social memory, 3-iodotyrosine (3-IY), DL-parachlorophenylalanine (PCPA), L-3,4-dihydroxyphenylalanine (L-DOPA), 5-L-hydroxytryptophan (5-HTP)

\section{INTRODUCTION}

Many animal species from a vast array of taxa can learn from others (i.e., social learning), particularly in the context of mate-choice (Avital and Jablonka, 2000; Danchin et al., 2004; Galef and Laland, 2005). Such observational learning can lead to mate-copying (Pruett-Jones, 1992), when females mate preferentially with a male showing similar characteristics as the male they saw being chosen by another female (trait-based copying, Bowers et al., 2012).

In Drosophila melanogaster, females are able to perform mate-copying (Mery et al., 2009) after watching only a single live demonstration of one female copulating with a male of a given phenotype and one male of another phenotype being rejected (Dagaeff et al., 2016; Danchin et al., 2018; Nöbel et al., 2018).

Despite some promising studies, research about the mechanisms of social learning in general and observational social learning in particular are still at the beginning (Burke et al., 2010; Debiec and Olsson, 2017; Kavaliers et al., 2017; Allsop et al., 2018). While social learning mechanisms are poorly known in any organism, D. melanogaster with its mini yet highly 
structured brain $(100,000$ cells) is one of the most favorable model species to dissect the neuronal processes of learning. Mostly, studies focused on simple kinds of learning tasks, where flies can learn from their own experience (non-social learning task), that are easier to standardize and historically well studied, like olfactory or visual associative learning (Quinn et al., 1974; Vogt et al., 2014, 2016; Cognigni et al., 2018). Thus, while the mechanisms of non-social learning in Drosophila are now welldescribed, the neurotransmitters and neural structures involved in observational social learning in Drosophila are unknown.

The formation of non-social associative memory requires dopamine in D. melanogaster: during the olfactory or visual learning process, it mediates aversive or appetitive unconditional stimuli (Riemensperger et al., 2005, 2011; Aso et al., 2012; Burke et al., 2012; Liu et al., 2012; Vogt et al., 2014), while serotonin is required for aversive place memory (Sitaraman et al., 2008), and for olfactory learning and memory (Johnson et al., 2011; Lee et al., 2011). Based on the fact that visual and olfactory learning share common neurotransmitters and neural structures (Vogt et al., 2014), we hypothesized that our model of observational social learning, mate-copying, involves the same two neurotransmitters. To address this, we used a pharmacological approach to reduce dopamine or serotonin synthesis with specific inhibitors of the limiting-step-enzyme of the synthetic pathway: 3-iodotyrosine (3-IY) inhibits tyrosine hydroxylase that catalyzes L-DOPA formation from tyrosine, and DL-para-chlorophenylalanine (PCPA) inhibits tryptophan hydroxylase that catalyzes 5-HTP formation from tryptophan, respectively. Young sexually mature virgin females were fed one of these drugs and their mate-copy ability was tested after a single demonstration. To ensure specificity of the drugs, we also had two rescue treatments in which the female received the drug (3-IY or PCPA) together with the immediate precursor of the neurotransmitter (L-DOPA or 5-HTP, respectively), so that the level of dopamine or serotonin was less reduced than with 3-IY or PCPA alone.

\section{MATERIALS AND METHODS}

\section{Fly Maintenance}

Wild-type Canton-S flies were raised in $30 \mathrm{ml}$ food vials containing standard corn flour-yeast-agar medium. The room was maintained at $25 \pm 0.8^{\circ} \mathrm{C}, 60 \pm 3 \%$ humidity, with a $12 \mathrm{~h}: 12 \mathrm{~h}$ light:dark cycle. Virgin flies were collected daily for the experiments and sexed without anesthesia, by gentle aspiration using a glass pipette, tubing and gauze. Flies were then kept in single-sex groups in food vials until the experiment started. As D. melanogaster females are reluctant to re-mate (Chapman et al., 2003), each female was used only once as demonstrator or observer.

\section{Drug Treatment}

The solutions were freshly prepared every week in vehicle (sucrose $5 \%$ in mineral water Vittel ${ }^{\circledR}$ ) and $200 \mathrm{ml}$ were poured on a Kimwipe paper $(1.5 \mathrm{~cm} \times 3.5 \mathrm{~cm})$ deposited in a $15 \mathrm{ml}$ Falcon tube. Nine 1-day-old virgin females were introduced in the tube for the length of the treatment, at $18^{\circ} \mathrm{C}, 12 \mathrm{~h}: 12 \mathrm{~h}$ light:dark cycle. To explore dopamine effect, flies were fed with 3 -IY $(10 \mathrm{mg} / \mathrm{ml}$, Sigma I8250) and/or L-DOPA (1 mg/ml, Sigma D9628) for 36-40 h (Bainton et al., 2000; Seugnet et al., 2008). To explore serotonin effect, flies were fed with PCPA $(10 \mathrm{mg} / \mathrm{ml}$, Sigma C6506) and/or 5-HTP (16 mg/ml, Sigma H9772) for 3 days, with papers being changed once during the 3 days period (Dierick and Greenspan, 2007; Plaçais et al., 2012). We used a high 5HTP concentration (30\% more than in Dierick and Greenspan, 2007) to ensure rescued serotonin levels in PCPA-treated flies in our conditions. This concentration did not affect mate-copying in flies fed with 5-HTP (Figure 2). The treatment "vehicle" consisted of vehicle solution given during 36-40 h or 3 days.

\section{Mate-Copying Experiment}

Flies were tested 3-4 days after eclosion. Experiments were conducted in the same conditions as fly maintenance. We used the same tubes set-up (double plastic tube $(0.8 \times 3 \mathrm{~cm}$ each) separated by a thin glass partition) and the speed-learning protocol as described in Dagaeff et al. (2016) except that matechoice tests were run either immediately to test learning, or $3 \mathrm{~h}$ $20 \pm 15 \mathrm{~min}$ after the demonstration, a time when associative memory in drosophila is composed of consolidated and labile memories (Folkers et al., 1993), two memories with independent pathways (Isabel et al., 2004; Scheunemann et al., 2012) so that we could detect a learning and/or memory defect. Artificial male phenotypes were obtained by randomly dusting them in green or pink (neutral trait) using colored powders (green: Shannon Luminous Materials, Inc., \#B-731; red: BioQuip Products, Inc., \#1162R). Demonstrations in tube set-ups showed a demonstrator female choosing between the two male phenotypes while the treated female could observe through a transparent partition. After the end of the copulation of demonstrator flies, each observer female was either directly tested or placed individually in a food vial until the test. The mate-choice test then involved two new virgin green and pink males placed in a tube with the observer female. Time when courtship began (first wing vibration) and color of the male, as well as time when copulation started and color of the chosen male were recorded.

\section{Mate-Copying Index}

Observer females that chose the same male color as the demonstrator for copulation (copied) were given a mate-copying score of 1 , and females that chose the opposite color were given a score of 0 . A mate-copying index (MCI) was calculated as the mean mate-copying score per treatment. Samples in which only one male courted the female before she initiated mating were not used for the analysis of the mate-copying performance because only when both males showed their interest the female was unambiguously in a position of choice. Samples in which no copulation occurred after $30 \mathrm{~min}$ were excluded from the analyses.

\section{Ethics Statement}

Behavioral observations of $D$. melanogaster required no ethical approval and complied with French laws regarding animal welfare. We kept the number of flies used in this study as small as 
possible. We handled flies by gentle aspiration without anesthesia to minimize damage and discomfort. After the experiments, individuals were euthanized in a freezer.

\section{Analyses}

Mate-copying scores were analyzed with the $\mathrm{R}$ software 3.4.0 ( $\mathrm{R}$ Core Team, 2017). For each treatment, the difference from random choice was tested with a binomial test. For global comparisons, mate-copying scores were analyzed in a generalized linear mixed model (GLMM) with binary logistic regression (package lme4, Bates et al., 2014). A random block effect introduced into the models accounted for the non-independence of observer flies from the same block of 6 demonstrations and tests. The significance of fixed effects was tested using Wald chisquare tests implemented in the ANOVA function of the car package (Fox and Weisberg, 2011). Starting models included treatment, air pressure at the time of the test, and its variation within the 6 preceding hours, and interactions between these effects. We used a backward selection approach using $P$-values, removing the highest order interaction as soon as it was nonsignificant. The final model was always chosen as the one with the lowest Akaike Information Criteria (AIC, Akaike, 1969). Two-bytwo comparisons between treatments were done using post hoc $X^{2}$ tests.

\section{RESULTS}

\section{PCPA and 3-IY Impair Learning or Memory in Mate-Copying}

We first tested whether females' mate-copying performance was affected after a PCPA or a 3-IY treatment. We analyzed the mate-copying scores (Figure 1) and found that females fed with the vehicle mate-copied, while females lacking serotonin or dopamine did not. We then compared the three groups and found a significant difference (GLMM, $N=241, X^{2}=7.26$, $P=0.027$ ), which we also found when comparing PCPA- or 3-IY-treated flies to the vehicle (Figure 1 and Supplementary Table S1). Thus, PCPA and 3-IY impaired mate-copying in these conditions. We also measured courtship duration in each group and found no statistical difference between them (Supplementary Figure S1).

\section{Dopamine and Serotonin Are Both Required for Learning in a Mate-Copying Context}

We then tested female mate-copying immediately after the demonstration, in order to study learning capacities only, and not memory retention. To ensure that the mate-copying defects observed in Figure 1 depend on lacking dopamine or serotonin, and not to a side-effect of the drug, we added four more treatments: PCPA with 5-HTP, 5-HTP, 3-IY with L-DOPA and L-DOPA. We measured mate-copying scores in all groups (Figure 2) and found that all groups copied except PCPA and 3IY treated females. We compared mate-copying scores in the five groups that copied and found no statistical difference (GLMM,
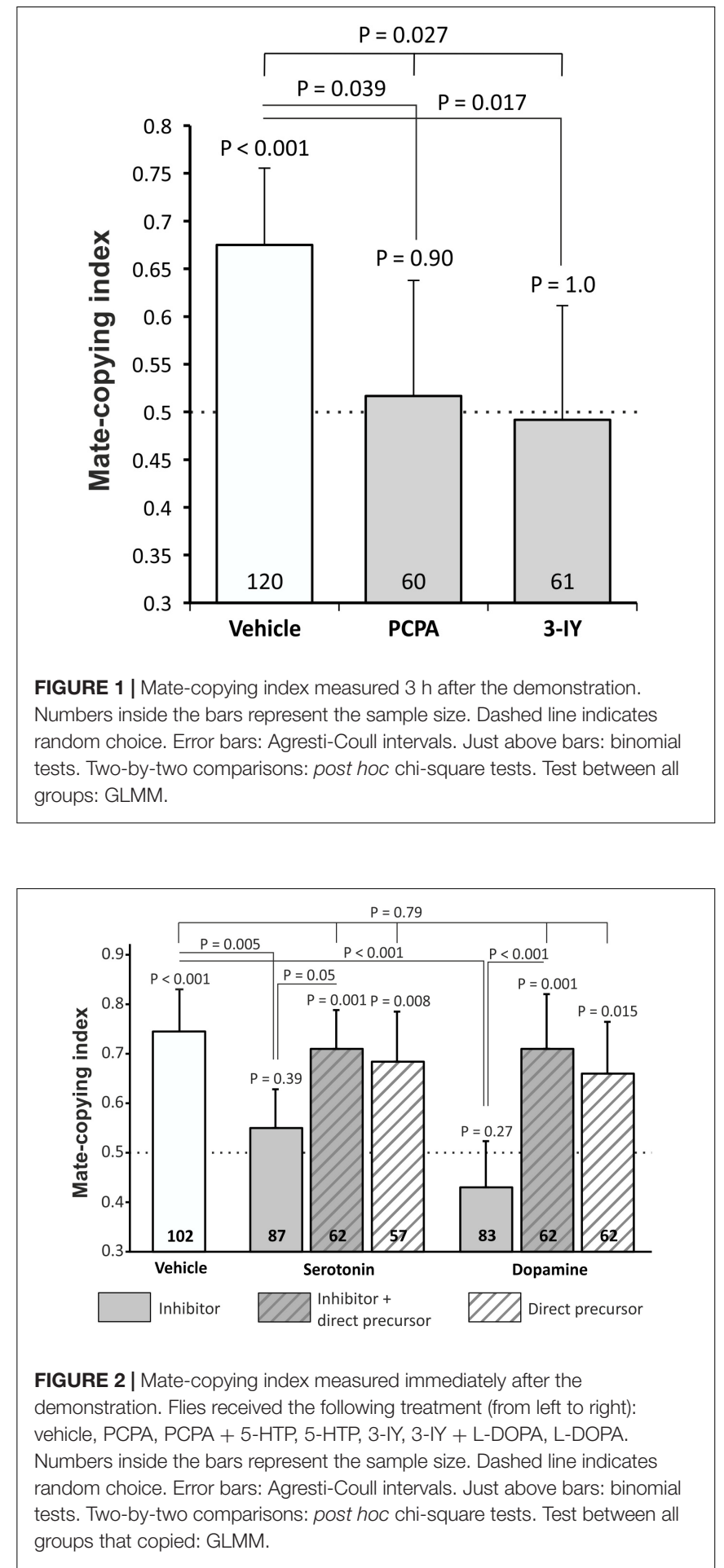

$N=345, X^{2}=1.72, P=0.79$ ), indicating that 5-HTP and L-DOPA given alone did not alter mate-copying ability, and could rescue mate-copying in females treated with the inhibitor. Additionally, we found a significant difference between inhibitortreated flies and flies fed with the vehicle or rescued flies (Figure 2 and Supplementary Table S2). Thus, flies lacking dopamine 
or serotonin are not able to learn a mate preference from the demonstration.

\section{DISCUSSION}

We found that dopamine and serotonin are both required in learning during mate-copying. Observer females lacking these neurotransmitters were unable to learn the successful male phenotype in the demonstration while control females receiving the vehicle solution, females treated with 5-HTP or L-DOPA, and females treated with the precursor together with the inhibitor copied the choice of the demonstrator immediately after the demonstration. This is in accordance with other studies showing that dopamine and serotonin are required for learning. In an olfactory learning task, dopamine is required to mediate the unconditional stimulus after a single training phase (Riemensperger et al., 2011; Aso et al., 2012; Burke et al., 2012; Liu et al., 2012; Lin et al., 2014; Vogt et al., 2014). Alterations in behavioral tracking were reported in flies lacking dopamine (Andretic et al., 2005), but dopamine-deficient flies were shown to have no alteration in visual perception and display a normal electroretinogram (Riemensperger et al., 2011). Thus, the defects we observed are not due to deficient vision, although we cannot exclude attention deficiency in dopaminedepleted flies. Serotonin is necessary to form place memory (Sitaraman et al., 2008) and associative olfactory learning memory (Johnson et al., 2011; Lee et al., 2011). Mate-copying can be compared to associative learning with pairing between a conditional and an unconditional stimulus (Avarguès-Weber et al., 2015): the conditional stimulus would be the color of the male copulating with the demonstrator female while the unconditional reinforcing stimulus could be the observation of the copulation. Under these circumstances, dopamine would mediate the reinforcing stimulus. Our results provide one more indication that the pathways underlying memory formation are comparable for visual social information and for olfactory information, and it was shown that both share mushroom body circuits for memory consolidation (Vogt et al., 2014). Matecopying was also described in many vertebrates (Dugatkin and Godin, 1993; White and Galef, 1999; Waynforth, 2007; Galef et al., 2008), so the mechanistic results discovered in Drosophila could be a starting point for such studies in vertebrates, as many vertebrate pathways and genes have homologs in Drosophila.

We showed that dopamine and serotonin are both required in mate-copying. This result paves the way for further studies of the neural pathways underlying social observational learning in D. melanogaster. The next step is now to dig into the role of each

\section{REFERENCES}

Akaike, H. (1969). Fitting autoregressive models for prediction. Ann. Inst. Stat. Math. 21, 243-247. doi: 10.1007/BF0253 2251

Allsop, S. A., Wichmann, R., Mills, F., Burgos-Robles, A., Chang, C.-J., FelixOrtiz, A. C., et al. (2018). Corticoamygdala transfer of socially derived information gates observational learning. Cell 173, 1329.e18-1342.e18. doi: $10.1016 /$ j.cell.2018.04.004 of these neurotransmitters, by assessing the neural structures and the receptors involved in this social learning task.

\section{DATA AVAILABILITY}

The raw data supporting the conclusions of this manuscript will be made available by the authors, without undue reservation, to any qualified researcher.

\section{AUTHOR CONTRIBUTIONS}

MM carried out the experiments, performed the analyses, and drafted the manuscript. SN contributed in the writing of the manuscript. ED and GI designed the experiments and jointly supervised all steps in the process. All authors gave final approval for publication.

\section{FUNDING}

This work was supported by the "Laboratoires d'Excellence (LABEX)" TULIP (ANR-10-LABX-41), the Toulouse Initiative of Excellence "IDEX UNITI" (ANR11-IDEX-0002-02) transversality grant, and the "MoleCulture" project from Agence Nationale pour la Recherche grant (ANR-18-CE37-0015) to GI and ED. ED and SN were also supported by the Soc- $\mathrm{H}^{2}$ ANR project (ANR-13-BSV7-0007-01) to ED. MM's salary was provided by a grant from the French ministry of higher education and research. SN's salary was provided by Soc- $\mathrm{H}^{2}$, a Marie Curie PRESTIGE grant (PRESTIGE-2014-1-0005) and received support from the ANR-Labex Institute for Advanced Study in Toulouse. GI benefited from a CNRS Excellence Chair.

\section{ACKNOWLEDGMENTS}

We would like to thank Nathalie Parthuisot and Tristan Lafont Rapnouil for help in fly care, Audrey Dussutour for providing food production equipment, and three referees for their valuable comments.

\section{SUPPLEMENTARY MATERIAL}

The Supplementary Material for this article can be found online at: https://www.frontiersin.org/articles/10.3389/fnbeh. 2018.00334/full\#supplementary-material

Andretic, R., van Swinderen, B., and Greenspan, R. J. (2005). Dopaminergic modulation of arousal in Drosophila. Curr. Biol. 15, 1165-1175. doi: 10.1016/ j.cub.2005.05.025

Aso, Y., Herb, A., Ogueta, M., Siwanowicz, I., Templier, T., Friedrich, A. B., et al. (2012). Three dopamine pathways induce aversive odor memories with different stability. PLoS Genet. 8:e1002768. doi: 10.1371/journal.pgen.100 2768

Avarguès-Weber, A., Lihoreau, M., Isabel, G., and Giurfa, M. (2015). Information transfer beyond the waggle dance: observational learning 
in bees and flies. Front. Ecol. Evol. 3:24. doi: 10.3389/fevo.2015. 00024

Avital, E., and Jablonka, E. (2000). Animal Traditions: Behavioural Inheritance in Evolution. Cambridge: Cambridge University Press. doi: 10.1017/CBO9780511542251

Bainton, R. J., Tsai, L. T.-Y., Singh, C. M., Moore, M. S., Neckameyer, W. S., and Heberlein, U. (2000). Dopamine modulates acute responses to cocaine, nicotine and ethanol in Drosophila. Curr. Biol. 10, 187-194. doi: 10.1016/S0960-9822(00) 00336- 5

Bates, D., Mächler, M., Bolker, B., and Walker, S. (2014). Fitting Linear MixedEffects Models using lme4. arXiv [preprint]. arXiv:1406.5823

Bowers, R. I., Place, S. S., Todd, P. M., Penke, L., and Asendorpf, J. B. (2012). Generalization in mate-choice copying in humans. Behav. Ecol. 23, 112-124. doi: 10.1093/beheco/arr164

Burke, C. J., Huetteroth, W., Owald, D., Perisse, E., Krashes, M. J., Das, G., et al. (2012). Layered reward signalling through octopamine and dopamine in Drosophila. Nature 492, 433-437. doi: 10.1038/nature11614

Burke, C. J., Tobler, P. N., Baddeley, M., and Schultz, W. (2010). Neural mechanisms of observational learning. Proc. Natl. Acad. Sci. U.S.A. 107, 1443114436. doi: 10.1073/pnas.1003111107

Chapman, T., Bangham, J., Vinti, G., Seifried, B., Lung, O., Wolfner, M. F., et al. (2003). The sex peptide of Drosophila melanogaster: female post-mating responses analyzed by using RNA interference. Proc. Natl. Acad. Sci. U.S.A. 100, 9923-9928. doi: 10.1073/pnas.1631635100

Cognigni, P., Felsenberg, J., and Waddell, S. (2018). Do the right thing: neural network mechanisms of memory formation, expression and update in Drosophila. Curr. Opin. Neurobiol. 49, 51-58. doi: 10.1016/j.conb.2017. 12.002

Dagaeff, A.-C., Pocheville, A., Nöbel, S., Loyau, A., Isabel, G., and Danchin, E. (2016). Drosophila mate copying correlates with atmospheric pressure in a speed learning situation. Anim. Behav. 121, 163-174. doi: 10.1016/j.anbehav. 2016.08.022

Danchin, E., Nöbel, S., Pocheville, A., Dagaeff, A.-C., Demay, L., Alphand, M., et al. (2018). Cultural flies: conformist social learning in fruit flies predicts longlasting mate-choice traditions. Science 362, 1025-1030. doi: 10.1126/science. aat 1590

Danchin, É, Giraldeau, L.-A., Valone, T. J., and Wagner, R. H. (2004). Public information: from nosy neighbors to cultural evolution. Science 305, 487-491. doi: 10.1126/science.1098254

Debiec, J., and Olsson, A. (2017). Social fear learning: from animal models to human function. Trends Cogn. Sci. 21, 546-555. doi: 10.1016/j.tics.2017. 04.010

Dierick, H. A., and Greenspan, R. J. (2007). Serotonin and neuropeptide F have opposite modulatory effects on fly aggression. Nat. Genet. 39, 678-682. doi: $10.1038 / \mathrm{ng} 2029$

Dugatkin, L. A., and Godin, J.-G. J. (1993). Female mate copying in the guppy (Poecilia reticulata): age-dependent effects. Behav. Ecol. 4, 289-292. doi: 10. 1093/beheco/4.4.289

Folkers, E., Drain, P., and Quinn, W. G. (1993). Radish, a Drosophila mutant deficient in consolidated memory. Proc. Natl. Acad. Sci. U.S.A. 90, 8123-8127. doi: $10.1073 /$ pnas.90.17.8123

Fox, J., and Weisberg, S. (2011). An $\{R\}$ Companion to Applied Regression. 2nd Edn. Thousand Oaks, CA: Sage Publishing.

Galef, B. G., and Laland, K. N. (2005). Social learning in animals: empirical studies and theoretical models. Bioscience 55, 489-499. doi: 10.1641/0006-3568(2005) 055[0489:SLIAES]2.0.CO;2

Galef, B. G., Lim, T. C. W., and Gilbert, G. S. (2008). Evidence of mate choice copying in Norway rats, Rattus norvegicus. Anim. Behav. 75, 1117-1123. doi: 10.1016/j.anbehav.2007.08.026

Isabel, G., Pascual, A., and Preat, T. (2004). Exclusive consolidated memory phases in Drosophila. Science 304, 1024-1027. doi: 10.1126/science.1094932

Johnson, O., Becnel, J., and Nichols, C. D. (2011). Serotonin receptor activity is necessary for olfactory learning and memory in Drosophila melanogaster. Neuroscience 192, 372-381. doi: 10.1016/j.neuroscience.2011. 06.058

Kavaliers, M., Matta, R., and Choleris, E. (2017). Mate-choice copying, social information processing, and the roles of oxytocin. Neurosci. Biobehav. Rev. 72, 232-242. doi: 10.1016/j.neubiorev.2016.12.003
Lee, P.-T., Lin, H.-W., Chang, Y.-H., Fu, T.-F., Dubnau, J., Hirsh, J., et al. (2011) Serotonin-mushroom body circuit modulating the formation of anesthesiaresistant memory in Drosophila. Proc. Natl. Acad. Sci. U.S.A. 108, 13794-13799. doi: 10.1073/pnas.1019483108

Lin, S., Owald, D., Chandra, V., Talbot, C., Huetteroth, W., and Waddell, S. (2014). Neural correlates of water reward in thirsty Drosophila. Nat. Neurosci. 17, 1536-1542. doi: 10.1038/nn.3827

Liu, C., Plaçais, P.-Y., Yamagata, N., Pfeiffer, B. D., Aso, Y., Friedrich, A. B., et al. (2012). A subset of dopamine neurons signals reward for odour memory in Drosophila. Nature 488, 512-516. doi: 10.1038/nature11304

Mery, F., Varela, S. A. M., Danchin, É, Blanchet, S., Parejo, D., Coolen, I., et al. (2009). Public versus personal information for mate copying in an invertebrate. Curr. Biol. 19, 730-734. doi: 10.1016/j.cub.2009.02.064

Nöbel, S., Danchin, E., and Isabel, G. (2018). Mate-copying for a costly variant in Drosophila melanogaster females. Behav. Ecol. 29, 1150-1156. doi: 10.1093/ beheco/ary095

Plaçais, P.-Y., Trannoy, S., Isabel, G., Aso, Y., Siwanowicz, I., Belliart-Guérin, G., et al. (2012). Slow oscillations in two pairs of dopaminergic neurons gate long-term memory formation in Drosophila. Nat. Neurosci. 15, 592-599. doi: 10.1038/nn.3055

Pruett-Jones, S. (1992). Independent versus nonindependent mate choice: do females copy each other? Am. Nat. 140, 1000-1009. doi: 10.1086/285452

Quinn, W. G., Harris, W., and Benzer, S. (1974). Conditioned Behavior in Drosophila melanogaster. Proc. Natl. Acad. Sci. U.S.A. 71, 708-712. doi: 10.1073/ pnas.71.3.708

R Core Team (2017). R: A Language and Environment for Statistical Computing. Vienna: R Foundation for Statistical Computing.

Riemensperger, T., Isabel, G., Coulom, H., Neuser, K., Seugnet, L., Kume, K., et al. (2011). Behavioral consequences of dopamine deficiency in the Drosophila central nervous system. Proc. Natl. Acad. Sci. U.S.A. 108, 834-839. doi: 10.1073/ pnas.1010930108

Riemensperger, T., Völler, T., Stock, P., Buchner, E., and Fiala, A. (2005). Punishment prediction by dopaminergic neurons in Drosophila. Curr. Biol. 15, 1953-1960. doi: 10.1016/j.cub.2005.09.042

Scheunemann, L., Jost, E., Richlitzki, A., Day, J. P., Sebastian, S., Thum, A. S., et al. (2012). Consolidated and labile odor memory are separately encoded within the Drosophila brain. J. Neurosci. 32, 17163-17171. doi: 10.1523/jneurosci.3286-12. 2012

Seugnet, L., Suzuki, Y., Vine, L., Gottschalk, L., and Shaw, P. J. (2008). D1 Receptor activation in the mushroom bodies rescues sleep-loss-induced learning impairments in Drosophila. Curr. Biol. 18, 1110-1117. doi: 10.1016/ j.cub.2008.07.028

Sitaraman, D., Zars, M., LaFerriere, H., Chen, Y.-C., Sable-Smith, A., Kitamoto, T., et al. (2008). Serotonin is necessary for place memory in Drosophila. Proc. Natl. Acad. Sci. U.S.A. 105, 5579-5584. doi: 10.1073/pnas.0710168105

Vogt, K., Aso, Y., Hige, T., Knapek, S., Ichinose, T., Friedrich, A. B., et al. (2016). Direct neural pathways convey distinct visual information to Drosophila mushroom bodies. eLife 5:e14009. doi: 10.7554/eLife.14009

Vogt, K., Schnaitmann, C., Dylla, K. V., Knapek, S., Aso, Y., Rubin, G. M., et al. (2014). Shared mushroom body circuits underlie visual and olfactory memories in Drosophila. eLife 3:e02395. doi: 10.7554/eLife.02395

Waynforth, D. (2007). Mate choice copying in humans. Hum. Nat. 18, 264-271. doi: $10.1007 / \mathrm{s} 12110-007-9004-2$

White, D. J., and Galef, B. G. Jr. (1999). Mate choice copying and conspecific cueing in Japanese quail, Coturnix coturnix japonica. Anim. Behav. 57, 465-473. doi: 10.1006/anbe.1998.1015

Conflict of Interest Statement: The authors declare that the research was conducted in the absence of any commercial or financial relationships that could be construed as a potential conflict of interest.

Copyright (c) 2019 Monier, Nöbel, Danchin and Isabel. This is an open-access article distributed under the terms of the Creative Commons Attribution License (CC BY). The use, distribution or reproduction in other forums is permitted, provided the original author(s) and the copyright owner(s) are credited and that the original publication in this journal is cited, in accordance with accepted academic practice. No use, distribution or reproduction is permitted which does not comply with these terms. 